\section{THE EFFECT OF COMORBIDITY AND GENDER ON IN- HOSPITAL MORTALITY IN PATIENTS WITH FALLS: RESULTS FROM A LARGE ASIAN TERTIARY CARE HOSPITAL}

${ }^{1,2}$ Yong Yang, ${ }^{3}$ Emily Ho, ${ }^{2,4}$ Julian Thumboo, ${ }^{2}$ Truls Østbye, ${ }^{5}$ Yee Sien $\mathrm{Ng}^{6}{ }^{6} \mathrm{Chern}$ Hui Jeffrey Fong, ${ }^{2,7}$ Kok Yong Fong. 'Department of Epidemiology, Medical Board, Singapore General Hospital, Outram Road, Singapore 169608; ${ }^{2}$ Duke-NUS Graduate Medical School, 8 College Road, Singapore 169857; ${ }^{3}$ Department of Endocrinology, Singapore General Hospital, Outram Road, Singapore 169608; ${ }^{4}$ Department of Rheumatology and Immunology, Singapore General Hospital, Outram Road, Singapore 169608; ${ }^{5}$ Department of Rehabilitation Medicine, Singapore General Hospital, Outram Road, Singapore 169608; ${ }^{6}$ Healthcare Analytics, Integrated Health Information Systems (IHIS), SingHealth Corporate Office, 6 Serangoon North Ave 5, Singapore 554910; ${ }^{7}$ Medical Board, Singapore General Hospital, Outram Road, Singapore 169608

\subsection{6/injuryprev-2016-042156.500}

Background Falls and fall-related injuries are a major public health issue among older adults worldwide. Causes and prognostic indicators of death in older patients with fall are lacking in Asian countries, especially in local older population. We aimed to assess the disease burden of fall and the usefulness of Charlson comorbidity index (CCI) and male sex as risk adjusted hospital mortality predictors in older Asian patients with fall using hospital administrative database. Methods: Historical cohort study of hospital discharge database from 1 January 2004 to 30 June 2015 to identify cases with fall and comorbidity using International Statistical Classification of Diseases and Related Health Problems, $9^{\text {th }}$ Revision, Australian Modification (ICD-9/10-AM) codes.

Results Over the study period, 16346 patients met the criteria of fall with hospital mortality rate of $4.3 \%$. The hospital mortality rates $(2.3 \%, 4.9 \%, 6.5 \%$, and $10.9 \%$ respectively, $\mathrm{P}<0.001)$ and hospital length of stay (geometric mean, 5.3, 6.1, 7.3, and 6.7 days, respectively, $\mathrm{P}<0.001$ ) increased consistently for patients with CCI ranging from none, low, moderate to high grade, respectively. Logistic regression model analysis showed that CCI (odds ratio, OR 5.7 high vs. none, $\mathrm{p}<0.001$ ) and male sex (OR 2.1, p < 0.001) were significant and independent predictors of hospital mortality. Similar results were also seen with hospital length of stay by zero-truncated negative binomial regression model analysis. Conclusion: The burden of fall is high in this older Asian population. Comorbidities and male sex were some of the most important contributors to hospital mortality and resource utilisation.

\section{CAN THE PROVISION OF A HOME HELP SERVICE FOR THE ELDERLY POPULATION REDUCE THE INCIDENCE OF FALL-RELATED INJURIES?}

\footnotetext{
${ }^{1,2}$ Carl Bonander, ${ }^{1,2}$ Johanna Gustavsson, ${ }^{1,2}$ Finn Nilson. 'Division of Risk Management, Department of Environmental and Life Sciences, Karlstad University, Sweden; ${ }^{2}$ Centre for Public Safety, Karlstad University, Sweden

\subsection{6/injuryprev-2016-042156.501}

Background Fall-related injuries are a global public health problem, especially in elderly populations. In this study, the effect of an intervention aimed at reducing the risk of falls in the homes of community-dwelling elderly persons was evaluated. The intervention, which involves home hazards reduction by providing a minor home help service, is provided in the majority of Swedish municipalities.
}

Methods Intention-to-treat effect estimates were derived using quasi-experimental time series intervention (ITS) analysis for immediate effects and a difference-in-discontinuity (RD) design for long term effects, and community-level estimates were pooled using meta-analysis. The outcome measure was the incidence of fall-related hospitalizations in the treatment population, the age of which varied by municipality ( $\geq 65$ years, $\geq 67$ years, $\geq 70$ years or $\geq 75$ years).

Results We found no statistically significant reductions in injury incidence in the ITS (IRR 1.01 [95\% CI: 0.98-1.05]) or RD (IRR 1.00 [95\% CI: 0.97-1.03]) analyses. The results are robust to several different model specifications, including segmented panel regression analysis with linear trend change and community fixed effects parameters.

Conclusions It is unclear whether absence of an effect is due to a low efficacy of the home hazards modifications provided, or a result of low utilisation. Additional studies of the effects on other quality of life measures are recommended before conclusions are drawn regarding the cost-effectiveness of the provision of home help services.

\section{PREVENTION OF ACCIDENTAL FALLS AMONG OLDER PEOPLE IN FINLAND - NATIONAL IKINÄ-PROGRAM}

${ }^{1}$ Satu Pajala, ${ }^{1}$ Laura Kolehmainen, ${ }^{1}$ Riitta Koivula, ${ }^{2}$ Mirka Råback, ${ }^{1}$ Anne Lounamaa. ${ }^{1}$ National Institute for Health and Welfare, Finland; ${ }^{2}$ Independent Researcher, Finland

\subsection{6/injuryprev-2016-042156.502}

Background In Finland, more than 900 older people $65+$ died due the fall in 2012. In addition, a fall was contributing factor of death of 770 persons 70 years and older. In 2012, costs of hospital inpatient care due to falls were close to EUR 400 million (THL injury database 2014).

Description of the problem Falls prevention has become extremely timely along with Finnish ageing policy prioritising older people living at their own home as long as possible. Despite vast quantity of evidence about falls prevention, the actions to put them in practice have not been as systematic and widespread as possible. Falls prevention needs to be an integral part of care and services for older people at all levels of care and among all service providers.

Results National program, called IKINÄ, was launched in 2006 to enhance dissemination and implementation of sustainable falls prevention in Finland. Aim of the program is to provide knowledge and stress the importance of falls prevention to policy-makers and other executive authorities in national level as well as municipalities. To enhance implementation of falls prevention IKINÄ-program produces evidence-based tools and materials for professionals and organisations working with older people.

During past ten years awareness of falls, their consequences and need for falls prevention have increased among professionals, both at executive level and those working in clinical practice, in the field of older people care and services in Finland. For example, several social and health care organisations have set the reduction of falls and fall injuries as their strategic goals. Thus, implementation of evidence-based falls prevention has become more systematic.

Conclusions Falls prevention requires to be continuous work and a joint effort of professionals working at all levels of care system of older people services. Sufficient resources should be allocated for falls prevention to ensure safe living at home for older people. 